\title{
Safety and Efficacy of Cosmetic Augmentation of the Nasal Tip and Nasal Dorsum With Expanded Polytetrafluoroethylene A Randomized Clinical Trial
}

Yifei Gu, MD; Wenxin Yu, PhD, MD; Yunbo Jin, PhD, MD; Hui Chen, PhD, MD; Gang Ma, PhD, MD; Shih-jen Chang, MD; Xiaoxi Lin, PhD, MD

IMPORTANCE Expanded polytetrafluoroethylene (ePTFE) is a widely used alloplast, but studies of augmentation of the nasal tip and nasal dorsum with ePTFE are lacking.

OBJECTIVE To investigate whether attaching the conchal cartilage as shield grafts at the distal end of the graft can prevent extrusion and whether the use of ePTFE in the nasal tip can achieve a stable aesthetic outcome.

DESIGN, SETTING, AND PARTICIPANTS A randomized clinical trial was performed from April 1, 2006, to October 31, 2008. Follow-up was completed in 2012 and 2017. The study was conducted at the Department of Plastic and Reconstructive Surgery, Shanghai Ninth People's Hospital, Shanghai Jiao Tong University School of Medicine, Shanghai, China. One hundred fifty patients seeking rhinoplasty were recruited, and 129 were eligible for inclusion in the study. All the recruited patients were intent-to-treat populations who were randomized into 2 groups: an ePTFE-only group and an ePTFE with conchal cartilage group.

INTERVENTIONS Expanded PTFE was used for augmentation of the nasal tip and nasal dorsum in both groups. Conchal cartilage was placed above the distal end in the ePTFE with conchal cartilage group.

MAIN OUTCOMES AND MEASURES Preoperative and postoperative standard photographs were obtained. Postoperative satisfaction and complications were evaluated.

RESULTS Of the 129 patients (mean age [range], 28 years [21-45 years]; 6 male and 123 female) eligible for inclusion in this study, 76 (mean age [range], 31 years [22-45 years]; 4 male and 72 female) were available for follow-up (mean [range], 106.9 months [100-131 months]) through 2017, including 39 in the ePTFE-only group and 37 in the ePTFE with conchal cartilage group. Infection occurred in 2 patients (5\%) in the ePTFE-only group vs 1 patient (3\%) in the ePTFE with conchal cartilage group (infection rate [OR, 1.946; 95\% Cl, $0.169-22.413 ; P>.99])$. Soft-tissue reaction occurred in 0 patients in the ePTFE-only group vs 1 patient (3\%) in the ePTFE with conchal cartilage group (soft-tissue reaction rate [OR, $0.000 ; 95 \% \mathrm{Cl}, 0.000-8.538 ; P=.49]$ ). Irregularity occurred in 0 patients in the ePTFE-only group vs 3 patients ( $8 \%$ ) in the ePTFE with conchal cartilage group (irregularity rate [OR, $0.000 ; 95 \% \mathrm{Cl}, 0.000-1.066 ; P=.11])$. Extrusion occurred in neither of the 2 groups.

CONCLUSIONS AND RELEVANCE A new technique using ePTFE alone for augmentation of the nasal tip and nasal dorsum achieved safe, attractive, and acceptable outcomes in nasal contouring and patient satisfaction. The use of conchal cartilage as a shield in the nasal tip is not necessary.

LEVEL OF EVIDENCE 1.

TRIAL REGISTRATION Chinese Clinical Trial Registry identifier: ChiCTR-INR-17011101

JAMA Facial Plast Surg. 2018;20(4):277-283. doi:10.1001/jamafacial.2017.2423

Published online February 22, 2018.
Supplemental content

+ CME Quiz at

jamanetwork.com/learning and CME Questions page 344
Author Affiliations: Department of Plastic and Reconstructive Surgery, Shanghai Ninth People's Hospital, Shanghai Jiao Tong University School of Medicine, Shanghai, China.

Corresponding Author: Xiaoxi Lin, MD, PhD, Department of Plastic and Reconstructive Surgery, Shanghai Ninth People's Hospital, Shanghai Jiao Tong University School of Medicine, 639 Zhizaoju Rd, Shanghai 200011, China(linxiaoxi@126.com). 
C ompared with noses of persons of European descent, the Asian nose typically has a low, flat nasal dorsum and insufficient nasal tip projection, thick lobular skin, wide lobules, abundant subcutaneous fatty tissues, alar flaring, a retracted columella, and a small osteocartilaginous framework. ${ }^{1}$ Consequently, surgery of the nasal tip projection along the dorsal augmentation is the most requested procedure among Asian patients. Autologous cartilage is considered by many surgeons to be the most desirable graft material. In Asian patients, an insufficient amount of septal and conchal cartilages for rhinoplasty is commonly observed, and the use of rib cartilage is related to potential donor site morbidity and warping. ${ }^{2}$ For decades, alloplastic implants have been particularly favored in Asian countries for augmentation in rhinoplasty because of their availability, ease of contouring, and lack of donor site morbidity. Silicone implants and expanded polytetrafluoroethylene (ePTFE) are the most widely used alloplast. ${ }^{3,4}$ Alloplastic materials are generally noted to be suitable for nasal dorsum augmentation, but their placement at the nasal tip (mostly silicone implants) can lead to various problems. These include skin thinning, erythema, transparency, tip deformation, implant extrusion, and compression of the cartilaginous framework. ${ }^{5}$ The silicone implant slightly affects the length and projection of the nasal tip. Before the present randomized clinical trial, we had operated on a number of patients using ePTFE in augmentation of the nasal tip and nasal dorsum. All patients exhibited an aesthetic improvement in the shape of the nasal tip, and the complication rate did not markedly increase; such is not the case when a silicone implant is used. Therefore, we aimed to investigate the longterm safety and efficacy of this technique, specifically regarding (1) whether attaching the conchal cartilage as shield grafts at the distal end of the graft can prevent extrusion and (2) whether the use of ePTFE in the nasal tip can achieve a stable aesthetic outcome. After a 10-year follow-up, we present our views, describe our technique, and propose an alternative method for rhinoplasty.

\section{Methods}

A prospective randomized clinical trial of patients undergoing rhinoplasty of the nasal tip and nasal dorsum by ePTFE implantation with or without conchal cartilage as a shield graft was performed from April 1, 2006, to October 31, 2008 (Figure 1). The study was conducted at the Department of Plastic and Reconstructive Surgery, Shanghai Ninth People's Hospital, Shanghai Jiao Tong University School of Medicine, Shanghai, China. The trial protocol is available in the Supplement. All surgical procedures were performed by one of us (X.L.) using the same technique and material. The study was approved by the institutional review board of the Shanghai Ninth People's Hospital and adhered to the tenets of the Declaration of Helsinki. Written informed consent was obtained from each participant.

The patients were recruited and then randomized into 2 study groups, an ePTFE-only group and an ePTFE with conchal cartilage group. All the recruited patients were intent-to-

\section{Key Points}

Question Is it safe and efficacious to use expanded polytetrafluoroethylene at the nasal tip and nasal dorsum for cosmetic augmentation?

Findings In this randomized clinical trial of 76 patients, complication rates were low and there were no significant differences between the expanded polytetrafluoroethylene-only group and the expanded polytetrafluoroethylene with conchal cartilage group.

Meaning Use of expanded polytetrafluoroethylene alone for augmentation of the nasal tip and nasal dorsum is safe and efficacious; use of conchal cartilage as a shield in the nasal tip is not necessary.

treat populations. The method used to generate the random allocation sequence was according to that described by Yu et al. ${ }^{6}$ Only ePTFE was used in patients assigned odd numbers. Alloplast and conchal cartilage were used in patients assigned even numbers. Preoperative and postoperative standard photographs were obtained. Postoperative satisfaction and complications, including infection, soft-tissue reaction, extrusion, and irregularity, were evaluated. Postoperative follow-up was completed in 2012 and 2017.

\section{Surgical Technique}

All procedures were performed with local anesthesia using 1\% lidocaine mixed with 1:100 000 epinephrine. Harvesting of conchal cartilage was conducted using a retroauricular approach. We noted the border and avoided damaging the morphologic features of the auricular pavilion. An inverted-V transcolumellar incision with infracartilaginous incisions was made to expose the entire osseocartilaginous framework. The whole nasal skin-soft-tissue pocket was dissected carefully and extensively to reduce the tension on the alloplast and lengthen the nose. The lower lateral cartilages were not dissected because they are needed to protect the implant from the sagging that potentially results from the stabilizing force of the soft-tissue envelope. The L-shaped ePTFE (Tisuthes; Shanghai Suokang Medical Implants Co) alloplast was shaped on a small sterile table with a fresh blade and new gloves. An ePTFE implant was tailored to the desired shape according to the needs of the patient. Typically, the filling thickness of the dorsum measured approximately 2 to $5 \mathrm{~mm}$ and could be increased to approximately 3 to $6 \mathrm{~mm}$ at the hump if required. We sutured the ePTFE patch to the original implant to increase the thickness if the patient required a prominent nasal dorsum. Near the tip part, the implant was tailored to an appropriate thickness. Meanwhile, the strut part of the L-shaped ePTFE implant was shaved to a length of approximately $1 \mathrm{~cm}$ and then sutured to the lobular part by using 5-0 polydioxanone. Both procedures aimed to increase the projection of the tip part to provide definition of a new tip.

If harvested, conchal cartilage was trimmed into a single-layer shield graft and placed above the caudal border of the lobular part to cover and protect the nasal tip. The 
alloplast was carefully vacuum impregnated in a bacteriostatic solution before it was inserted into the dissected dorsal pocket. ${ }^{7}$ The position and plane of the alloplast were verified to ensure that no curves or folds were present. In ePTFE placement, a no-touch technique was applied as much as possible to reduce the possibility of contamination. Precise placement was necessary to ensure a pleasing cosmetic result and to avoid surface irregularities or step-off deformities. The edges were tapered to create smooth round corners. Neither a temporary transcutaneous suture at the cephalic part nor a permanent suture with the media crura at the caudal part was necessary to secure the ePTFE position. All incisions were closed using 6-0 polypropylene sutures. Nasal and auditory packing was used and then removed within 24 hours. Postoperative oral systemic antibiotics were routinely prescribed for 3 days (Figure 2).

\section{Statistical Analysis}

The descriptive statistics of the study population were analyzed by frequency for categorical variables and by mean (SD) for normally distributed continuous variables. Occurrence of complications in the 2 groups was compared using Fisher exact test. Two-sided $P<.05$ was considered to be statistically significant. All analyses were performed using SPSS statistical software, version 19.0 (IBM Corporation).

\section{Results}

Of the 129 patients (mean age [range], 28 years [21-45 years]; 6 male and 123 female) recruited into this randomized clinical trial from April 1, 2006, to October 31, 2008, 76 (mean age [range], 31 years [22-45 years]), including 4 men and 72 women, were available for follow-up through March 2017. Thirty-nine patients received rhinoplasty with ePTFE implantation only (ePTFE-only group), and 37 received rhinoplasty with the conchal cartilage as shield grafts above the ePTFE at the nasal tip (ePTFE with conchal cartilage group). A total of 66 patients $(87 \%)$ had primary cases, and the other 10 patients (13\%) had revision cases after an unsuccessful rhinoplasty.
During a mean follow-up of 106.9 months (range, 100-131 months) through 2017, complications associated with ePTFE occurred in 4 of 76 patients (5\%), including 3 patients (4\%) with infection and 1 patient (1\%) with a temporary soft-tissue reaction. Two patients with infection were in the ePTFE-only group, and the remaining 1 patient with infection and 1 patient with soft-tissue reaction were in the ePTFE with conchal cartilage group. The patients with infection did not respond to antibiotic treatment and required implant removal. Infection occurred within 6 months after rhinoplasty in all 4 patients. One primary patient in the ePTFE with conchal cartilage group developed infection within 5 months after rhinoplasty, and a pustule gradually appeared and enlarged on her left nasal tip skin. A revision patient in the ePTFE-only group had poor healing of the right nostril incision within 1 month after rhinoplasty. Pus drained out of the incision when pinching the nasal tip (Figure 3). The remaining patient in the ePTFE with conchal

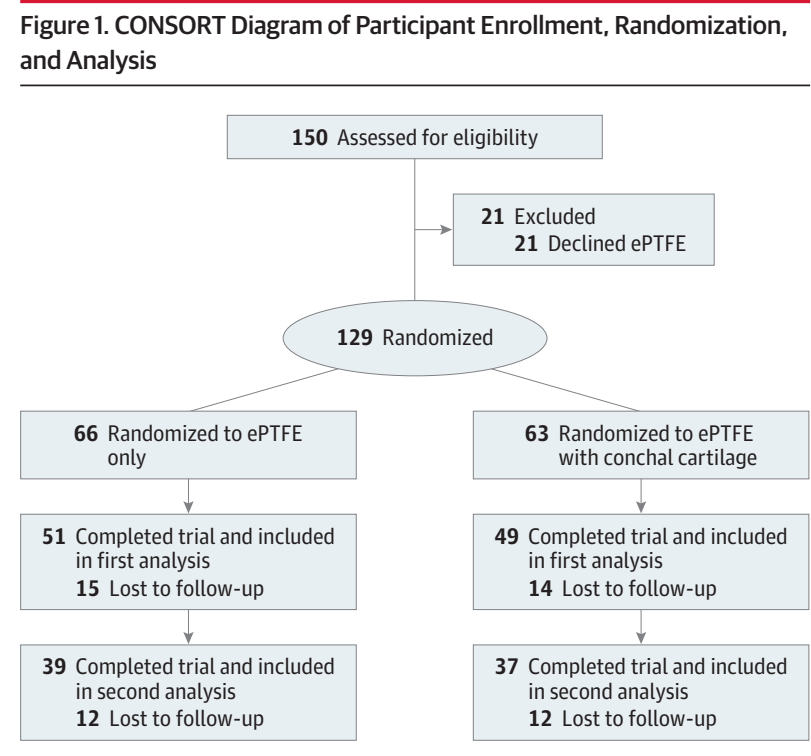

ePTFE indicates expanded polytetrafluoroethylene.

Figure 2. Shape and Position of Expanded Polytetrafluoroethylene (ePTFE)

A ePTFE implant

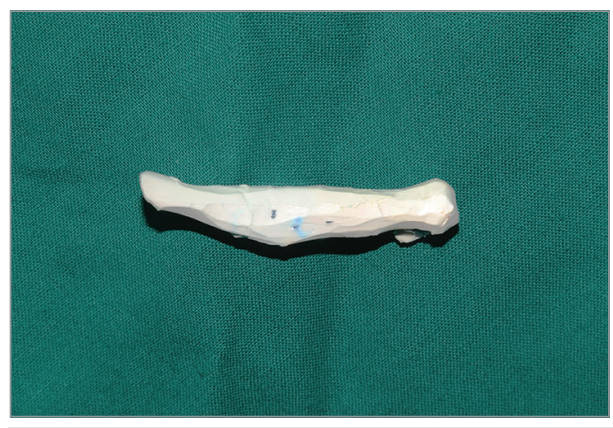

B Carved ePTFE implant

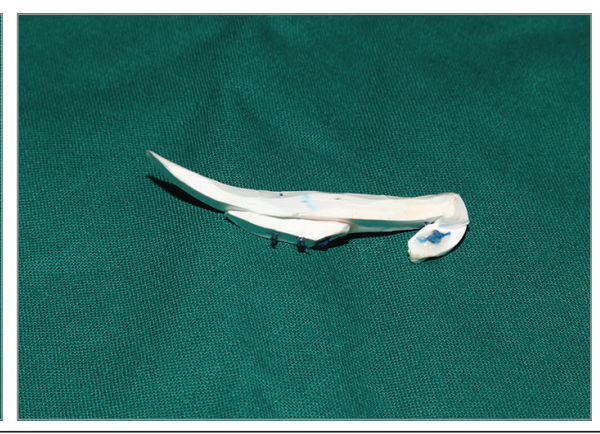

C Schematic diagram of the ePTFE position

A and B, The L-shaped ePTFE implant was carved to the desired shape. Shown is the same implant after carving with the different viewing angle. C, The schematic diagram was drawn by 2 of us (Y.G. and X.L.) and is published with permission. 


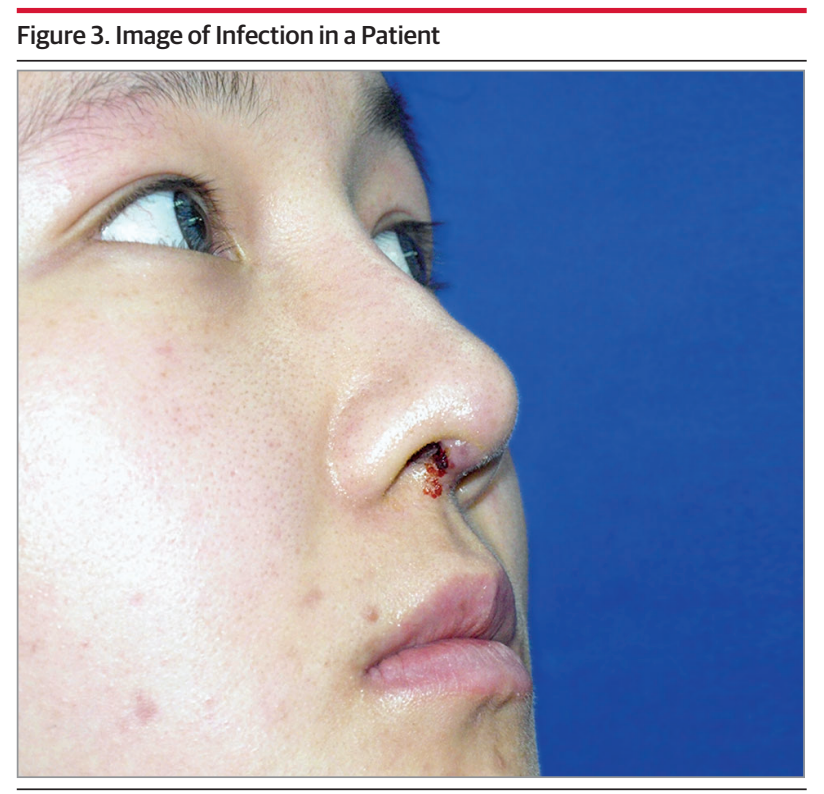

A revision patient in the expanded polytetrafluoroethylene-only group had poor healing of the right nostril incision within 1 month after rhinoplasty. Pus drained out of the incision when pinching the nasal tip.

cartilage group also experienced poor healing of the right nostril incision within 1 month after rhinoplasty. The implant was integrally removed through the original incision, and no further augmentation was permitted at the same stage. The patient with soft-tissue reaction had nasal skin redness immediately after surgery that lasted for 4 weeks and then involuted spontaneously.

The following measures were not significantly different between the 2 groups: overall complication rate (odds ratio [OR], 0.346; 95\% CI, 0.063-1.906; $P=.26$ ), constituent ratio of the complication rate $(P=.14)$, infection rate (OR, 1.946; 95\% CI, 0.169-22.413; $P>$.99), soft-tissue reaction rate (OR, 0.000; $95 \%$ CI, 0.000-8.538; $P=.49$ ), extrusion rate (OR cannot be calculated; $P>$.99), and irregularity rate (OR, 0.000; 95\% CI, 0.000$1.066 ; P=.11)$.

In the ePTFE with conchal cartilage group, 3 of the $37 \mathrm{pa}-$ tients $(8 \%)$ presented with palpability of the conchal cartilage graft with visible edges of the shield graft and underwent revision; none of the patients in the ePTFE-only group required further correction. There was no significant difference in complication rates between the 2 groups $(5.1 \%$ in the ePTFE-only group and $13.5 \%$ in the ePTFE with conchal cartilage group). No serious displacement was reported in either group. In addition to the 4 patients with infection and softtissue reaction, 4 other patients without infection were dissatisfied with their outcome. Two of these 4 patients requested implant removal. The remaining patients in the study obtained satisfactory results. At postoperative clinic visits, no problems were reported related to the position or texture of the implants. During clinical examinations, the alloplast appeared to be well integrated without any contour irregularities. We could not distinguish between the 2 groups based on the tip contour and softness by palpability. The nasal dorsum was significantly augmented after surgery. Enhanced tip pro- jection and downward rotation of the nasal tip were achieved (Figure 4). All nasal incisions healed well without obvious scar. The donor sites showed no disfigurement.

\section{Discussion}

Widely known as Gore-Tex (W. L. Gore Associates) in the West, ePTFE has been used clinically since it was introduced as a vascular prosthesis. ${ }^{8}$ Owing to the long-term efficacy, safety, and biocompatibility established in vascular surgery, ${ }^{9,10}$ ePTFE has been used extensively for abdominal wall defect, hernia, and facial reconstruction, ${ }^{11}$ as well as other indications. The material was first described in rhinoplasty augmentation by Rothstein and Jacobs ${ }^{12}$ in 1989 and was subsequently approved in 1993 by the US Food and Drug Administration for use in rhinoplasty and other aesthetic surgical procedures. Expanded PTFE is a polymer of carbon bound to fluorine formed into an inert weave of PTFE nodules and thin PTFE fibrils. ${ }^{13}$ It is a microporous alloplastic compound, with pore sizes ranging from 10 to $30 \mu \mathrm{m}$ (mean, $22 \mu \mathrm{m}$ ), that provides sufficient tissue ingrowth and fixation of the implant. Several animal models and clinical experience have confirmed the high biocompatibility and safety of ePTFE. ${ }^{14-17}$ Minimal chronic inflammation and foreign-body reaction were observed in those studies. A tiny fibrotic capsule forms around the implant. ${ }^{14-16}$ For our routine operations, we selected Tisuthes (Shanghai Suokang Medical Implants), a widely used ePTFE implant brand in China that can provide different models of shape and hardness.

To our knowledge, the present study is the first specialized, prospective randomized clinical trial to use ePTFE in augmentation of the nasal tip and nasal dorsum. Before this trial, we had accumulated years of experience using ePTFE alone to augment the nasal tip and nasal dorsum. Previous studies 7,18-2O $^{2}$ mainly focused on the safety and efficacy of using ePTFE in the nasal dorsum only; the infection rates in the previous studies ranged from $0 \%$ to $3.2 \%$. These results are similar to those obtained in the present study. Insufficient evidence was found to conclude that the application of ePTFE in the nasal tip may increase the infection rate during long-term follow-up.

Moreover, no patient in either group experienced alloplast extrusion during the 10-year follow-up. We concluded that the absence of extrusion was attributable to the alloplast not being applied as a columellar strut part. This clinical result contradicts the common view that the use of an alloplast in the nasal tip leads to negative outcomes. Direct contact between ePTFE and the soft tissue of the nasal tip did not predispose patients to complications, such as thinning of the overlying skin and implant exposure. Other researchers have asserted that volumetric compensation of smaller defects is more suitable than supportive functions. ${ }^{21}$ Adamson ${ }^{22}$ suggested the use of alloplast only for volume filling because extrusion rates are high when alloplast is used for structural support, ${ }^{5}$ which is unacceptable to both patients and physicians. Winkler et $\mathrm{al}^{23}$ reported that the highest exposure rate (23.4\%) was obtained when porous polyethylene (Medpor; Porex Technologies) was used as a columellar strut. Dong et $\mathrm{al}^{24}$ described a technique used in 1700 patients who underwent rhinoplasty with ePTFE 
Figure 4. Preoperative and Postoperative (After 4 Years) Clinical Images of Patients

A Preoperative frontal view in 2008

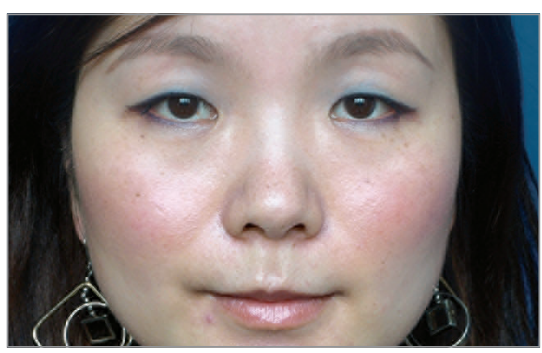

B Postoperative frontal view in 2012

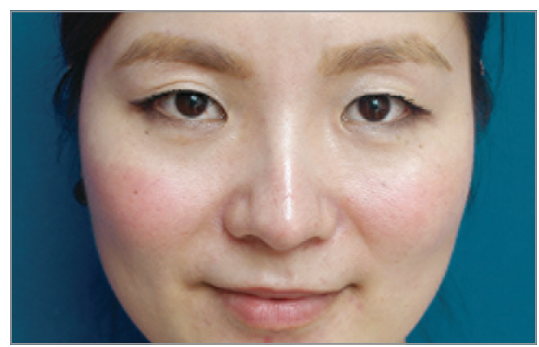

E Postoperative profile view in 2012
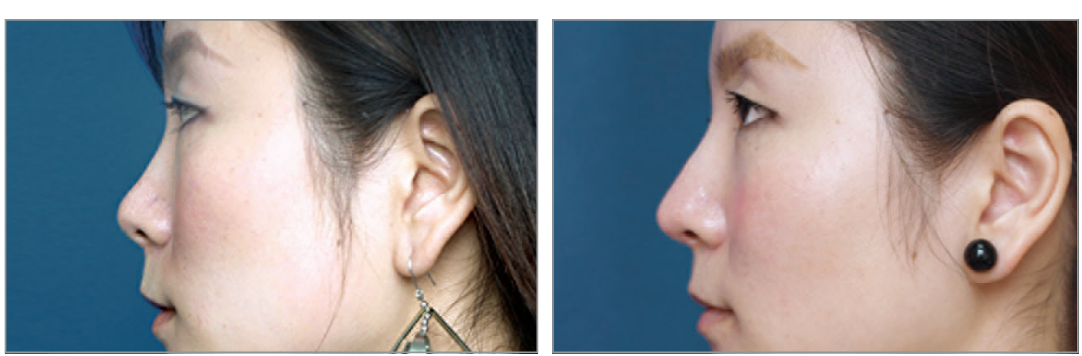

H Postoperative frontal view in 2012

G Preoperative frontal view in 2008
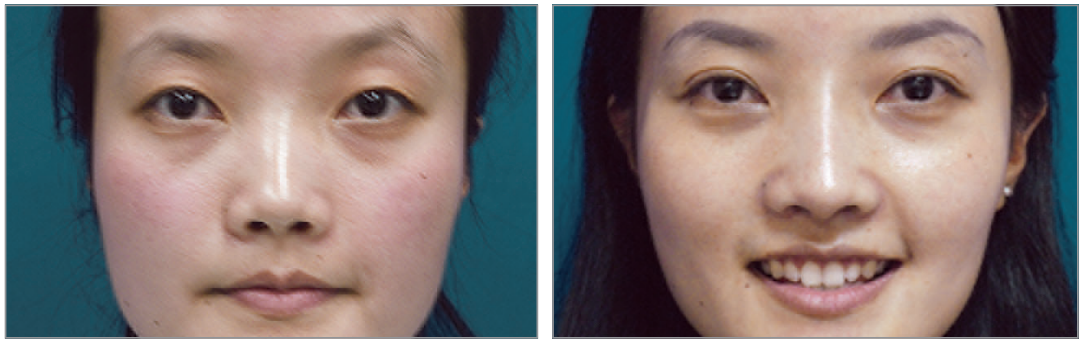

J Preoperative profile view in 2008

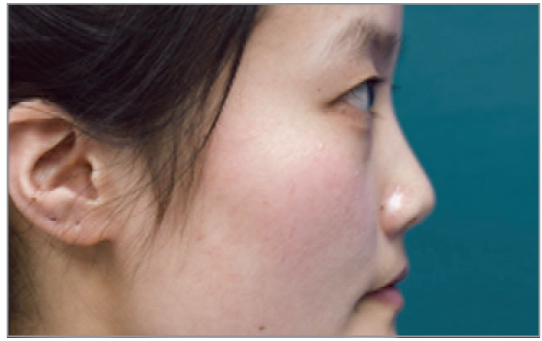

K Postoperative profile view in 2012

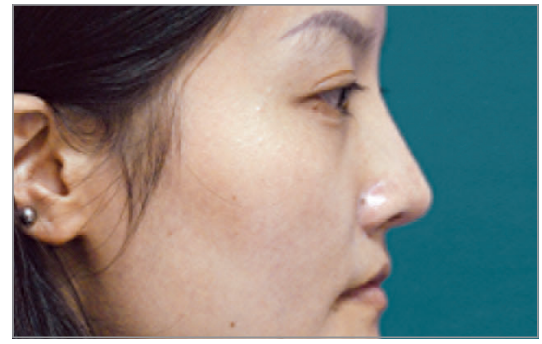

A-F, A patient in the expanded polytetrafluoroethylene (ePTFE)-only group. Compared with the preoperative anthropometric measurements, the postoperative nasal tip projection ratio increased from 0.67 to 0.73 , the nasolabial angle decreased from $127.94^{\circ}$ to $108.76^{\circ}$, and the columellar facial angle decreased from $118.54^{\circ}$ to $113.94^{\circ}$. G-L, A patient in the ePTFE with
I Postoperative frontal view in 2017

L Postoperative profile view in 2017

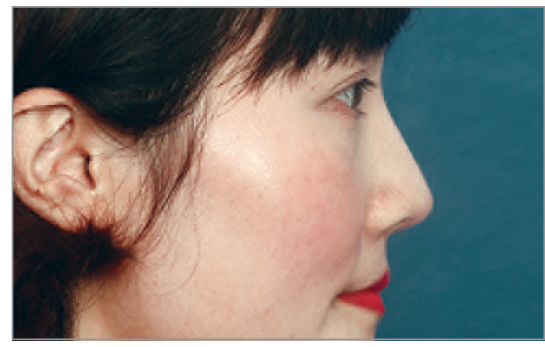

C Postoperative frontal view in 2017

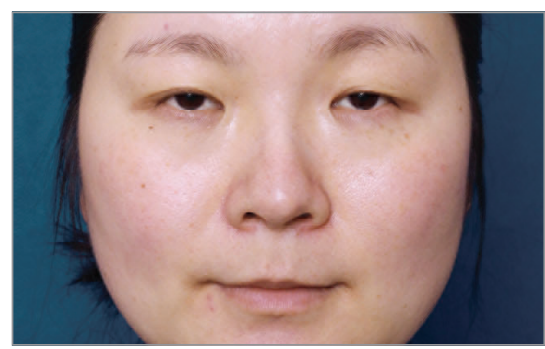

F Postoperative profile view in 2017
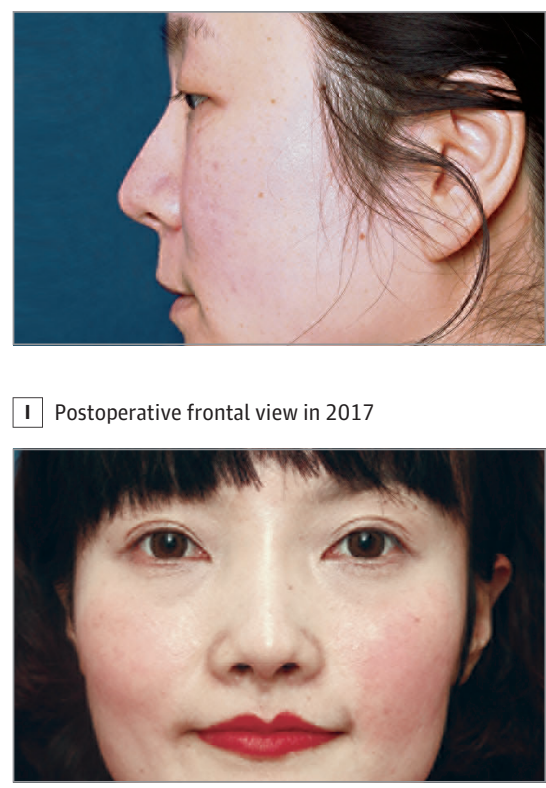

cartilage group. Compared with the preoperative anthropometric measurements, the postoperative nasal tip projection ratio increased from 0.55 to 0.64 , the nasolabial angle decreased from $109.54^{\circ}$ to $102.16^{\circ}$, and the columellar facial angle decreased from $117.30^{\circ}$ to $111.89^{\circ}$. as a combination of nasal filling and columellar strut grafts and found a 3\% prosthesis extrusion rate. Extrusion rates of silicone implants typically range from $2.1 \%$ to $3.7 \% .^{3}$ Lam and $\mathrm{Kim}^{25}$ used an L-shaped silicone implant to provide dorsal and tip augmentation. However, owing to the characteristics of silicone, the implant could appear to be unnatural and extruded if the columella portion of the implant was too pronounced.
A silicone implant needs to be shaved very thin to reduce the possibility of pressure necrosis on the tip skin and implant extrusion. The possibility of pliability and displacement is expected to increase ( $3 \%$ in the study by Lam and $\mathrm{Kim}^{25}$ ) even if subperiosteal fixation of the implant is performed. Lacking strong support, the projection of the nasal tip is limited and does not meet the general requirements of Asian patients. 
In the early stage of our prospective randomized clinical trial, 3 patients had palpability of the conchal cartilage graft with visible edges of a shield graft in the nasal tip and required revision. No similar complications occurred after increased attention was given to the surgical techniques of creating smooth and round corners at both the alloplast and the shield cartilage. We concluded that localized dysvascularity ${ }^{26}$ or hypertension after surgery caused the soft-tissue reaction that manifested skin redness of the nasal tip in 1 patient and considered that the spontaneous improvement 4 weeks later might have resulted from the revascularization of the damaged tissue or adaptation to high pressure.

As previously mentioned, a weak and small cartilaginous framework, in addition to thick skin, requires a rigid and strong structural support, which necessitates harvesting costal cartilage. Dorsal augmentation using diced conchal cartilage is limited by its shortcomings of material, inclusion resorption, and long duration of the procedure. ${ }^{27,28}$ Costal cartilage provides a sufficient supply of graft material necessary not only for structural support to allow tip rotation but also for dorsal augmentation. ${ }^{29}$ Concerns regarding additional obvious chest scarring that the inframammary fold may fail to hide discourage the use of costal cartilage among Asian patients. In addition to donor site morbidity, patients are also concerned about rigidity, warping, and resorption of the costal cartilage. One study ${ }^{30}$ concluded that dorsal augmentation with an autologous costal cartilage leads to similar aesthetic outcomes but with a higher complication rate compared with dorsal augmentation with ePTFE.

The septal extension graft is an effective technique in rhinoplasty because it can reshape the nasal tip; the septal cartilage is the most commonly used material. However, the septal cartilage is considerably weaker, thinner, and smaller in Asian patients, particularly those with short-nose deformities. ${ }^{31,32}$

Compared with other techniques in rhinoplasty, the use of ePTFE in the nasal tip and nasal dorsum requires less operative time (usually < 40 minutes). Given our experience, we generally carve the alloplast once or perform a slight revision depending on the nasal contour. It is inconclusive whether operative time is an independent risk factor for complications; some surgeons report that the complication rate increases significantly after 3 hours. ${ }^{33-35}$ Short operative time and minimal trauma of surgery improve patient comfort and allow for quick recovery. On the basis of our experience, ePTFE can be removed integrally and be replaced with autogenous cartilage grafts in a second stage if patients develop infection or are dissatisfied with the material.
We believe that the characteristics of the ePTFE material and the Asian nose contribute to the safety of the implant. Tissue incorporation, cell attachment, and capsule formation reduce the tendency of the ePTFE to deteriorate. Thicker sebaceous skin also acts as a strong wall to decrease tension over the implant ${ }^{36}$ and prevent extrusion. Based on the $0 \%$ extrusion rate in our clinical trial, the use of conchal cartilage as a shield in the nasal tip is not necessary. The use of ePTFE at the nasal tip is an intermediate state between volume filling and structural support. Traditionally, the columellar graft has contributed to nasal tip elongation, and the ePTFE in our proposed technique resembles a lever projecting the nasal tip. As previously mentioned, the biocompatibility associated with minimal chronic inflammation and foreign-body reaction surrounding ePTFE prevents its extrusion from the nasal tip. The structure aids in resisting compressive forces on the skin envelope and contractile forces for wound healing. ${ }^{37}$ Increased nasal tip support contributes to creating and maintaining the desired degree of tip projection and rotation. Such a method is suitable for patients with saddle nose or short-nose deformities because ePTFE can provide a robust support to resist skin contracture, which is crucial for lengthening the nose and increasing tip projection by caudal rotation of the tip. We also recommend the use of this technique in primary patients who require augmentation of the nasal tip and nasal dorsum without further donor site morbidity associated with harvesting cartilage. In revision rhinoplasty, patients who are dissatisfied with the aesthetic outcomes of silicone implants can also choose ePTFE after being informed of a possible infection rate increase. ${ }^{38}$ We have not used the method described herein of using ePTFE in the nasal dorsum and tip in white patients because of their different racial/ethnic nasal characteristics.

\section{Limitations}

The major limitation of our prospective randomized clinical trial is the withdrawal bias attributed to a decade of followup. This factor may have affected the complication rate. Another limitation is that no patient-reported outcome measure tool was used to assess patient satisfaction.

\section{Conclusions}

A new technique using ePTFE alone for augmentation of the nasal tip and nasal dorsum achieved safe and attractive outcomes in nasal contouring and obtained satisfactory patient results. The use of conchal cartilage as a shield in the nasal tip is not necessary.

\section{ARTICLE INFORMATION}

Accepted for Publication: November 30, 2017.

Published Online: February 22, 2018. doi:10.1001/jamafacial.2017.2423

Open Access: This Open Access article is distributed under the terms of the Creative Commons Attribution Noncommercial License (http:// creativecommons.org/licenses/by-nc/4.0/) which permits any noncommercial use, distribution, and reproduction in any medium, provided the original author(s) and the source are cited.

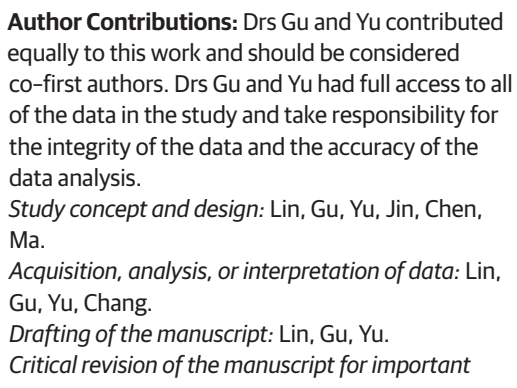

Author Contributions: Drs Gu and Yu contributed equally to this work and should be considered co-first authors. Drs Gu and Yu had full access to all of the data in the study and take responsibility for the integrity of the data and the accuracy of the data analysis.

Study concept and design: Lin, Gu, Yu, Jin, Chen, Ma.

Acquisition, analysis, or interpretation of data: Lin,

$\mathrm{Gu}, \mathrm{Yu}$, Chang.

Drafting of the manuscript: Lin, Gu, Yu.

Critical revision of the manuscript for important

intellectual content: All authors

Statistical analysis: Gu, Yu.

Administrative, technical, or material support: All authors.

Study supervision: Lin.

Conflict of Interest Disclosures: None reported.

Additional Contributions: We thank the patients for granting permission to publish this information. 


\section{REFERENCES}

1. Ahn JM. The current trend in augmentation rhinoplasty. Facial Plast Surg. 2006;22(1):61-69.

2. Neu BR. Segmental bone and cartilage reconstruction of major nasal dorsal defects. Plast Reconstr Surg. 2000;106(1):160-170.

3. Malone M, Pearlman S. Dorsal augmentation in rhinoplasty: a survey and review. Facial Plast Surg. 2015;31(3):289-294.

4. Tham C, Lai YL, Weng CJ, Chen YR. Silicone augmentation rhinoplasty in an Oriental population. Ann Plast Surg. 2005;54(1):1-5.

5. Warren RJ, Neligan PC. Plastic Surgery: Volume 2: Aesthetic Plastic Surgery. 3rd ed. Philadelphia, PA: Saunders Elsevier; 2012:174.

6. Yu W, Jin Y, Yang J, et al. Occurrence of bruise, hematoma, and pain in upper blepharoplasty using blunt-needle vs sharp-needle anesthetic injection in upper blepharoplasty: a randomized clinical trial. JAMA Facial Plast Surg. 2017;19(2):128-132.

7. Conrad K, Torgerson CS, Gillman GS. Applications of Gore-Tex implants in rhinoplasty reexamined after 17 years. Arch Facial Plast Surg. 2008;10(4):224-231.

8. Soyer T, Lempinen M, Cooper P, Norton L, Eiseman B. A new venous prosthesis. Surgery. 1972; 72(6):864-872.

9. McAuley CE, Steed DL, Webster MW. Seven-year follow-up of expanded polytetrafluoroethylene (PTFE) femoropopliteal bypass grafts. Ann Surg. 1984;199(1):57-60.

10. Singh S, Baker JL Jr. Use of expanded polytetrafluoroethylene in aesthetic surgery of the face. Clin Plast Surg. 2000;27(4):579-593.

11. Neel HB III. Implants of Gore-Tex. Arch Otolaryngol. 1983;109(7):427-433.

12. Rothstein SG, Jacobs JB. The use of Gore-Tex implants in nasal augmentation operations. Entechnology. 1989;68:40, 42, 44-45.

13. Boyce B. Physical characteristics of expanded-polytetrafluoroethylene grafts. In: Stanley JC, ed. Biologic and Synthetic Vascular Prosthesis. New York, NY: Grune \& Stratton; 1982: 5553-5561.

14. Durucu C, Kanlikama M, Mumbuc S, Bayazit $Y$, Bakir K, Karatas E. Medialization laryngoplasty with Gore-Tex: an animal study. J Voice. 2007;21(5):632639.
15. Schoenrock LD, Chernoff WG. Subcutaneous implantation of Gore-Tex for facial reconstruction. Otolaryngol Clin North Am. 1995;28(2):325-340.

16. Konior RJ. Facial paralysis reconstruction with Gore-Tex Soft-Tissue Patch. Arch Otolaryngol Head Neck Surg. 1992;118(11):1188-1194.

17. Kim JH, Park CH, Lee OJ, Lee JH, Hong SM. Histologic changes in transplanted expanded polytetrafluoroethylene in an animal model. Laryngoscope. 2012;122(1):17-22.

18. Owsley TG, Taylor CO. The use of Gore-Tex for nasal augmentation: a retrospective analysis of 106 patients. Plast Reconstr Surg. 1994;94(2):241-248.

19. Godin MS, Waldman SR, Johnson CM Jr. The use of expanded polytetrafluoroethylene (Gore-Tex) in rhinoplasty: a 6-year experience. Arch Otolaryngol Head Neck Surg. 1995;121(10):1131-1136.

20. Jin HR, Lee JY, Yeon JY, Rhee CS. A multicenter evaluation of the safety of Gore-Tex as an implant in Asian rhinoplasty. Am J Rhinol. 2006;20(6):615-619.

21. Lin G, Lawson W. Complications using grafts and implants in rhinoplasty. Oper Tech Otolaryngol-Head Neck Surg. 2007;18(4):315-323. Accessed January 11, 2018. doi:10.1016/j.otot.2007 09.004

22. Adamson PA. Grafts in rhinoplasty: autogenous grafts are superior to alloplastic. Arch Otolaryngol Head Neck Surg. 2000;126(4):561-562.

23. Winkler AA, Soler ZM, Leong PL, Murphy A, Wang TD, Cook TA. Complications associated with alloplastic implants in rhinoplasty. Arch Facial Plast Surg. 2012;14(6):437-441.

24. Dong L, Hongyu X, Gao Z. Augmentation rhinoplasty with expanded polytetrafluoroethylene and prevention of complications. Arch Facial Plast Surg. 2010;12(4):246-251.

25. Lam SM, Kim YK. Augmentation rhinoplasty of the Asian nose with the "bird" silicone implant. Ann Plast Surg. 2003;51(3):249-256.

26. Zelken JA, Hong JP, Chang CS, Hsiao YC. Silicone-polytetrafluoroethylene composite implants for Asian rhinoplasty. Ann Plast Surg. 2017; 78(2):131-137.

27. Kim JH, Jang YJ. Use of diced conchal cartilage with perichondrial attachment in rhinoplasty. Plast Reconstr Surg. 2015;135(6):1545-1553.
28. Tasman AJ. Advances in nasal dorsal augmentation with diced cartilage. Curr Opin Otolaryngol Head Neck Surg. 2013;21(4):365-371.

29. Park JH, Mangoba DC, Mun SJ, Kim DW, Jin HR. Lengthening the short nose in Asians: key maneuvers and surgical results. JAMA Facial Plast Surg. 2013;15(6):439-447.

30. Joo YH, Jang YJ. Comparison of the surgical outcomes of dorsal augmentation using expanded polytetrafluoroethylene or autologous costal cartilage. JAMA Facial Plast Surg. 2016;18(5):327-332.

31. Lin J, Chen X, Wang $X$, et al. A modified septal extension graft for the Asian nasal tip. JAMA Facial Plast Surg. 2013;15(5):362-368.

32. Kang JG, Ryu J. Nasal tip surgery using a modified septal extension graft by means of extended marginal incision. Plast Reconstr Surg. 2009;123(1):343-352.

33. Gordon NA, Koch ME. Duration of anesthesia as an indicator of morbidity and mortality in office-based facial plastic surgery: a review of 1200 consecutive cases. Arch Facial Plast Surg. 2006;8 (1):47-53.

34. Phillips BT, Wang ED, Rodman AJ, et al. Anesthesia duration as a marker for surgical complications in office-based plastic surgery. Ann Plast Surg. 2012;69(4):408-411.

35. Hardy KL, Davis KE, Constantine RS, et al. The impact of operative time on complications after plastic surgery: a multivariate regression analysis of 1753 cases. Aesthet Surg J. 2014;34(4):614-622.

36. Erlich MA, Parhiscar A. Nasal dorsal augmentation with silicone implants. Facial Plast Surg. 2003;19(4):325-330.

37. Hidalgo DA, Doft MA. The caudal septum columellar strut graft: an alternative for tip support. Plast Reconstr Surg. 2015;136(3):484-487.

38. Yap EC, Abubakar SS, Olveda MB. Expanded polytetrafluoroethylene as dorsal augmentation material in rhinoplasty on Southeast Asian noses: three-year experience. Arch Facial Plast Surg. 2011; 13(4):234-238. 